\title{
Equivalent stiffness of timber used in CLT: Closed-form estimates and numerical validation
}

\author{
Olivier Perret • Arthur Lebée • Cyril Douthe · Karam Sab
}

the date of receipt and acceptance should be inserted later

\begin{abstract}
In this paper, a closed-form approach is presented to estimate rapidly the equivalent stiffness of boards used in Cross Laminated Timber (CLT) panels from local orthotropic behavior at ring scale for varying sawing patterns.

It is first assumed that narrow edges are glued. In this case, closed-form Reuss and Voigt bounds are derived for the equivalent layer behavior of CLT. An application to Norway spruce boards is presented and reveals that the cross-layer (rolling) shear behavior lies between 100 and $150 \mathrm{MPa}$ with a careful selection of the board sawing pattern. Then, using finite element method, upper bounds for the cross-layer shear stiffness modulus of boards with and without glued edges are calculated and theoretical predictions are compared with recommendations and experimental data from the literature. Finally, it appears that these bounds remain relevant for CLT layer with unglued narrow edges for common aspect ratios.
\end{abstract}

\section{Introduction}

Cross-Laminated-Timber (CLT) consists of lumber layers stacked crosswise and glued on their lower and upper faces. Its quick and easy assembling, its low self-weight and its low environmental impact make this product competitive. In the last few years, several timber buildings made of CLT were built mainly in Northern America and in Western Europe such as Murray Grove in London (Lomholt, 2009) and the Treet in central Bergen (Mairs, 2016).

O. Perret · A. Lebée $\cdot$ C. Douthe $\cdot$ K. Sab

Laboratoire Navier UMR 8205 École des Ponts ParisTech, IFSTTAR, CNRS, Université Paris-Est, 6-8 Avenue Blaise Pascal, Cité Descartes, Champs-sur-Marne F- 77455 Marne-la-Vallée, France

E-mail: arthur.lebee@enpc.fr

olivier.perret@enpc.fr · cyril.douthe@ifsttar.fr·karam.sab@enpc.fr ORCID: 0000-0001-7865-6374
Nevertheless, the behavior of CLT panels is complex because of several heterogeneities at different scales. Here, only heterogeneities larger than the annual ring are considered. At ring scale, wood elastic behavior is generally modeled as an orthotropic material with three main directions: the longitudinal direction $L$ corresponding to wood fiber orientation, the radial and tangential directions $R$ and $T$. The fibers orientation leads to a strong stiffness and strength contrast between the stiff longitudinal direction and the soft radial and tangential directions. At board scale, another heterogeneity is due to the rotation of the material orthotropic coordinate system $(O, L, R, T)$ (Figure 1) where $O$ is located at the pith. The global orthotropic system of the board is defined as $(O, L, C, Z)$ where $C$ and $Z$ stand for cross and normal directions (Figure 1). The behavior of the board depends on the distribution of rings through the board section, called the sawing pattern. At layer scale, individual boards can be glued or not on their narrow edges depending on the manufacturing process. Free narrow edges lead to stress concentrations lowering the stiffness and strength of CLT panels under particular loads. Note that, for free narrow edges, self-contact may be ignored since there is a small gap between boards in practice. Finally, CLT are multi-layered panels strongly heterogeneous because of cross-wise orientations of layers.

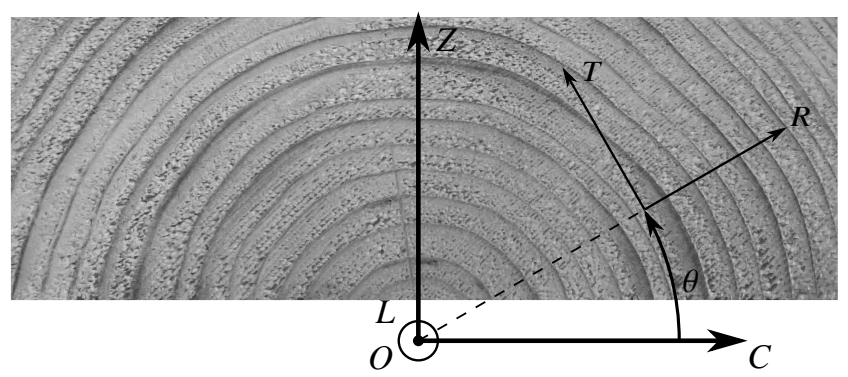

Fig. 1 Local and global orientations in a board 
From these observations, the mechanical behavior of CLT panels is difficult to model properly. The scales of heterogeneities cited above are not clearly distinct: the curvature radius and the thickness of annual rings are comparable with the thickness of boards, the board width is larger than the layer thickness, the number of layers in CLT is small (from three to eleven). Thus, these heterogeneities require careful attention when using homogenization techniques.

Nevertheless, in current recommendations for the design of CLT, panels are often modeled as multilayer plates or beams. This is the case of the $\gamma$-method recalled in Eurocode 5 (Eurocode 5-1-1, 2004, 2015) and adapted to CLT in the European Technical Agreement ATE-08/0271 for Stora Enso products where cross-layers are modeled as mechanical joints between longitudinal layers with a stiffness related to the equivalent cross-layer shear stiffness. In the CLT-designer software, Thiel and Schickhofer (2010) suggest to use Timoshenko beam theory and derived the shear stiffness of CLT beams from the Jourawski method. Advanced models have also been suggested by Franzoni et al (2016b) for the bending of CLT panels and by Perret et al (2016) for the buckling of CLT panels. In these methods, it is assumed that the mechanical behavior of each layer, composed of several boards, can be modeled as homogeneous. It is thus assumed that there exists an equivalent layer mechanical behavior which adequately takes into account the effect of lower scale heterogeneities such as ring distribution and narrow edges gaps.

This equivalent behavior is often based on an educated guess for a small collection of plate solicitations which emerge from practical applications. First, the longitudinal and cross Young's moduli $E_{L}$ and $E_{C}$ are involved in the bending stiffness for CLT floors (Kreuzinger, 1999; Gagnon and Pirvu, 2013; Thiel and Schickhofer, 2010) and in the in-plane stiffness for CLT walls (Thiel and Schickhofer, 2013; Thiel and Krenn, 2016; Perret et al, 2016). Because of the high stiffness contrast between $E_{L}$ and $E_{C}$, the cross Young's modulus $E_{C}$ is often neglected (Thiel and Schickhofer, 2010). Moreover, because of the thickness of CLT and of the cross layers, the out-of-plane shear stiffness of CLT, composed of the longitudinal shear stiffness modulus $G_{L Z}$ and of the cross-layer shear stiffness modulus $G_{C Z}$, is also involved in the bending (Franzoni et al, 2016b) and the buckling behavior of CLT (Perret et al, 2016). In the $\gamma$-method - detailed in (Gagnon and Pirvu, 2013) $-G_{L Z}$ is neglected because of the stiffness contrast compared to $G_{C Z}$. On the contrary, both $G_{L Z}$ and $G_{C Z}$ are involved in the shear analogy method - (Kreuzinger, 1999) also detailed in (Gagnon and Pirvu, 2013) - and the Timoshenko beam theory since the influence of $G_{L Z}$ is generally not negligible in CLT beams. Finally, the in-plane shear stiffness modulus $G_{L C}$ is involved in shear walls. Particularly, the narrow edge gluing and the board aspect ratio have a strong influence on $G_{L C}$ (Moosbrugger et al, 2006; Bogensperger et al, 2010; Franzoni et al,
2017). All these parameters necessitate recommendations depending on timber strength class, on the board aspect ratio and on the narrow edge gluing as suggested in the draft for a revised version of the Eurocode 5-1-1 (2015), but the sawing pattern may also be taken into account.

In this direction, Aicher et al (2001, 2016), Jakobs (2005) and Ehrhart et al (2015) investigated experimentally the effect of the sawing pattern on various equivalent layer stiffness moduli perpendicular to the grain and observed that this parameter has a strong influence on the mechanical behavior of the layer. Indeed, stress and strain fields are not uniform in the board because of the varying orientation of the wood material properties. This encouraged numerical parametric studies (Aicher and Dill-Langer, 2000; Jakobs, 2005; Nairn, 2007; Gustafsson and Danielsson, 2013; Aicher et al, 2016; Nairn, 2017). These investigations differ in the wood solicitation direction and the boundary conditions to apply to the board section in order to retrieve equivalent properties. However, they all rely on finite element simulations which may not be convenient for practical design.

In order to give a fast and reasonable estimate of the elastic behavior of CLT, closed-form expressions of the equivalent stiffness of a single board depending on its sawing pattern and the local elastic moduli are suggested in this paper. First, in Section 2.1, the equivalent stiffness is calculated from the average of the local stiffness and compliance matrix in all directions of the radial-tangential plane. Then, in Section 2.2, a finer estimation of the equivalent stiffness is established using Reuss lower bound and Voigt upper bound for a given sawing pattern. An application to Norway spruce boards with various sawing patterns is presented in Section 3 for boards with glued edges. In order to extrapolate these results to boards with free edges, the impact of edge gluing on the equivalent layer stiffness is first discussed in Section 4. Then, using finite element method, upper bounds for the cross-layer shear stiffness modulus $G_{C Z}$ of boards with and without glued edges are calculated and theoretical predictions are compared with recommendations and experimental data from the literature. Conclusions are finally drawn on the relevance of these bounds for the either analytical or numerical modeling of CLT panels.

\section{Closed-form expressions for the equivalent stiffness of a single board}

In this section, the equivalent stiffness of a single board is estimated from local behavior of wood using averaging techniques. It is based on the fundamental assumption that the applied average strain or stress is uniform at the scale of the board. The equivalent behavior of the board is expressed in the global orthotropic system $(O, L, C, Z)$ of the board (Figure 1). For doing so, wood variability is ignored as a first approach. Hence the annual rings are perfect concentric 
circles. It is also assumed that the local constitutive behavior of the wood in the frame $(O, L, R, T)$ is known and only dependent on the local orientation $\theta$ of the ring with respect to the frame $(O, L, C, Z)$. This excludes the distinction between earlywood and latewood which may have an influence on the equivalent stiffness behavior as pointed out in previous studies (Nairn, 2007). This also excludes the presence of juvenile wood with reduced mechanical properties close to the pith.

\subsection{Stiffness and compliance averages}

For a given board, the sawing pattern is not known a priori. A first approximation of the global behavior can be obtained by averaging the local behavior in all directions of the radialtangential plane $(O, R, T)$. For this purpose, the constitutive law between the local strain $\varepsilon$ and the local stress $\sigma$ is written using Kelvin's notation in the $(O, L, R, T)$ frame:

$$
\left(\begin{array}{c}
\varepsilon_{L} \\
\varepsilon_{R} \\
\varepsilon_{T} \\
\sqrt{2} \varepsilon_{R T} \\
\sqrt{2} \varepsilon_{L T} \\
\sqrt{2} \varepsilon_{L R}
\end{array}\right)=\left(\begin{array}{cccccc}
S_{11} & S_{12} & S_{13} & 0 & 0 & 0 \\
S_{12} & S_{22} & S_{23} & 0 & 0 & 0 \\
S_{13} & S_{23} & S_{33} & 0 & 0 & 0 \\
0 & 0 & 0 & S_{44} & 0 & 0 \\
0 & 0 & 0 & 0 & S_{55} & 0 \\
0 & 0 & 0 & 0 & 0 & S_{66}
\end{array}\right)\left(\begin{array}{c}
\sigma_{L} \\
\sigma_{R} \\
\sigma_{T} \\
\sqrt{2} \sigma_{R T} \\
\sqrt{2} \sigma_{L T} \\
\sqrt{2} \sigma_{L R}
\end{array}\right),
$$

where $S$ is the three-dimensional compliance matrix of the local orthotropic material. Considering local Young's moduli $E$, shear moduli $G$ and Poisson's ratio $v$, components of the three-dimensional compliance matrix $S$ are expressed as:

$$
\begin{gathered}
S_{11}=\frac{1}{E_{L}}, \quad S_{12}=-\frac{v_{L R}}{E_{L}}, S_{13}=-\frac{\nu_{L T}}{E_{L}}, \\
S_{22}=\frac{1}{E_{R}}, \quad S_{23}=-\frac{v_{R T}}{E_{R}}, \quad S_{33}=\frac{1}{E_{T}}, \\
S_{44}=\frac{1}{2 G_{R T}}, S_{55}=\frac{1}{2 G_{L T}}, S_{66}=\frac{1}{2 G_{L R}} .
\end{gathered}
$$

With Kelvin's notation, the rotation matrix $\boldsymbol{R}$ around longitudinal axis $L$ by an angle $\theta$ (Figure 1 ) is defined as:

$\boldsymbol{R}(\theta)=\left(\begin{array}{cccccc}1 & 0 & 0 & 0 & 0 & 0 \\ 0 & c^{2} & s^{2} & \sqrt{2} c s & 0 & 0 \\ 0 & s^{2} & c^{2} & -\sqrt{2} c s & 0 & 0 \\ 0 & -\sqrt{2} c s & \sqrt{2} c s & c^{2}-s^{2} & 0 & 0 \\ 0 & 0 & 0 & 0 & c & -s \\ 0 & 0 & 0 & 0 & s & c\end{array}\right)$

where $c=\cos (\theta)$ and $s=\sin (\theta)$. The compliance tensor $\boldsymbol{S}(\theta)$ after rotation by angle $\theta$ is given by:

$\boldsymbol{S}(\theta)=\boldsymbol{R}(\theta) \cdot \boldsymbol{S} \cdot{ }^{T} \boldsymbol{R}(\theta)$,

where ${ }^{T} \boldsymbol{R}$ is the transpose of $\boldsymbol{R}$.
The average of the compliance matrix $S(\theta)$ in the domain $\theta \in[0,2 \pi]$ gives a compliance matrix $\overline{\boldsymbol{S}}$ corresponding to a transversely isotropic material:

$\overline{\boldsymbol{S}}=\left(\begin{array}{cccccc}\bar{S}_{11} & \bar{S}_{12} & \bar{S}_{12} & 0 & 0 & 0 \\ \bar{S}_{12} & \bar{S}_{22} & \bar{S}_{22}-\bar{S}_{44} & 0 & 0 & 0 \\ \bar{S}_{12} & \bar{S}_{22}-\bar{S}_{44} & \bar{S}_{22} & 0 & 0 & 0 \\ 0 & 0 & 0 & \bar{S}_{44} & 0 & 0 \\ 0 & 0 & 0 & 0 & \bar{S}_{66} & 0 \\ 0 & 0 & 0 & 0 & 0 & \bar{S}_{66}\end{array}\right)$,

where

$$
\left\{\begin{array}{l}
\bar{S}_{11}=S_{11}, \\
\bar{S}_{12}=\frac{1}{2}\left(S_{12}+S_{13}\right), \\
\bar{S}_{22}=\frac{1}{8}\left[3\left(S_{22}+S_{33}\right)+2\left(S_{23}+S_{44}\right)\right], \\
\bar{S}_{44}=\frac{1}{4}\left(S_{22}+S_{33}+2\left(S_{44}-S_{23}\right)\right), \\
\bar{S}_{66}=\frac{1}{2}\left(S_{55}+S_{66}\right) .
\end{array}\right.
$$

This averaged compliance $\overline{\boldsymbol{S}}$ defines apparent moduli of a transversely isotropic elastic behavior. Let $E^{S}, G^{S}$ and $v^{S}$ denote the Young's moduli, shear moduli and Poisson's ratios from the averaged compliance. They are defined by the following expressions:

$$
\left\{\begin{aligned}
E_{L}^{S} & =\frac{1}{\bar{S}_{11}}=E_{L}, \\
E_{N}^{S} & =\frac{1}{\bar{S}_{22}}=8\left(\frac{3-v_{R T}}{E_{R}}+\frac{3-v_{T R}}{E_{T}}+\frac{1}{G_{R T}}\right)^{-1}, \\
v_{L N}^{S} & =-\frac{\bar{S}_{12}}{\bar{S}_{11}}=\frac{v_{L T}+v_{L R}}{2}, \\
G_{L N}^{S} & =\frac{1}{2 \bar{S}_{66}}=\left(\frac{1}{2 G_{L R}}+\frac{1}{2 G_{L T}}\right)^{-1}, \\
G_{N N}^{S} & =\frac{1}{2 \bar{S}_{44}}=2\left(\frac{1}{G_{R T}}+\frac{1+v_{R T}}{2 E_{R}}+\frac{1+v_{T R}}{2 E_{T}}\right)^{-1} .
\end{aligned}\right.
$$

Since there is no distinction here between cross and normal directions $C$ and $Z$ of the board, the notation $N$ is used for the normal directions of the board (perpendicular to the grain).

In the following, the three-dimensional stiffness matrix $\boldsymbol{C}(\theta)=\boldsymbol{S}^{-1}(\theta)$ is averaged in all directions of the radial tangential plane. Since Kelvin's notations are used here, the same rotation matrix $\boldsymbol{R}(\theta)$ is used to calculate the stiffness matrix $\boldsymbol{C}$ leading to similar results. The averaged stiffness matrix $\overline{\boldsymbol{C}}$ also corresponds to a transversely isotropic material:

$\overline{\boldsymbol{C}}=\left(\begin{array}{cccccc}\bar{C}_{11} & \bar{C}_{12} & \bar{C}_{12} & 0 & 0 & 0 \\ \bar{C}_{12} & \bar{C}_{22} & \bar{C}_{22}-\bar{C}_{44} & 0 & 0 & 0 \\ \bar{C}_{12} & \bar{C}_{22}-\bar{C}_{44} & \bar{C}_{22} & 0 & 0 & 0 \\ 0 & 0 & 0 & \bar{C}_{44} & 0 & 0 \\ 0 & 0 & 0 & 0 & \bar{C}_{66} & 0 \\ 0 & 0 & 0 & 0 & 0 & \bar{C}_{66}\end{array}\right)$,

where

$\left\{\begin{array}{l}\bar{C}_{11}=C_{11}, \\ \bar{C}_{12}=\frac{1}{2}\left(C_{12}+C_{13}\right), \\ \bar{C}_{22}=\frac{1}{8}\left[3\left(C_{22}+C_{33}\right)+2\left(C_{23}+C_{44}\right)\right], \\ \bar{C}_{44}=\frac{1}{4}\left(C_{22}+C_{33}+2\left(C_{44}-C_{23}\right)\right), \\ \bar{C}_{66}=\frac{1}{2}\left(C_{55}+C_{66}\right) .\end{array}\right.$ 
Note that the elastic behavior calculated from the stiffness average is not the same as the elastic behavior calculated from the compliance average: $\overline{\boldsymbol{C}} \neq \overline{\boldsymbol{S}}^{-1}$. From the stiffness matrix $\overline{\boldsymbol{C}}$, it is first necessary to compute $\overline{\boldsymbol{C}}^{-1}$ in order to identify engineering moduli as previously (7). Here, only the longitudinal shear stiffness modulus has a simple expression:

$G_{L N}^{C}=\frac{1}{2}\left(G_{L R}+G_{L T}\right)$.

2.2 Reuss lower bound and Voigt upper bound for a specific sawing pattern

One can then go further in the estimation of average properties, based on the geometry of boards. Indeed, assuming an equivalent layer with glued narrow edges, it is possible to derive bounds taking explicitly the board aspect ratio and the relative distance to the pith into account.

The Reuss lower bound compliance matrix $S^{R}$ is the average of the local compliance matrix $S(\theta)$, where $\theta$ is the ring orientation, over the full board section. Averaging the compliance matrix is equivalent to assume a uniform stress state $\sigma$ over the whole section. This lower bound is first derived for a centered board with respect to the pith (Figure 2) and then generalized to other configurations (Figure 4, 5 and 6).

\subsubsection{Reuss lower bound for a centered board}

For a rectangular board with the pith at the center (Figure 2), $S^{R}$ can be expressed as:

$S^{R}=\frac{1}{4 b t} \int_{-b}^{b} \int_{-t}^{t} \boldsymbol{S}(\theta) d C d Z$

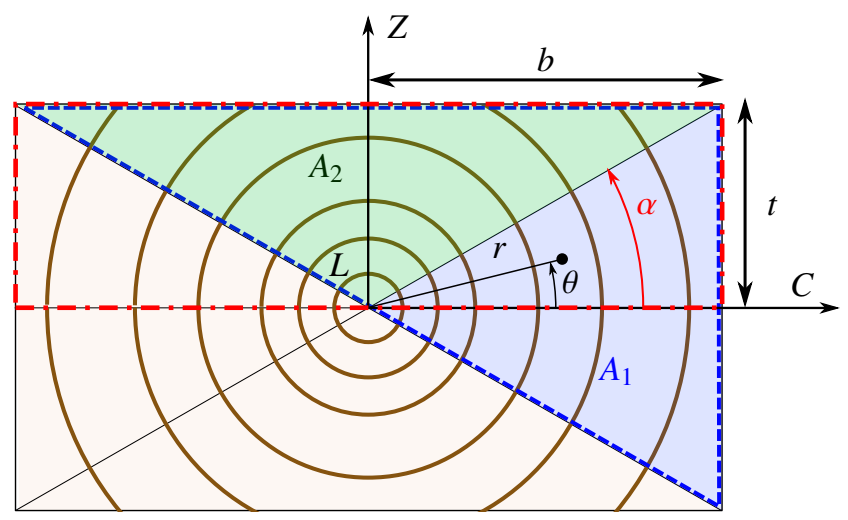

Fig. 2 Scheme of the board section

Note that for symmetry reasons, $\boldsymbol{S}(\theta)=\boldsymbol{S}(\pi+\theta)$. Hence, the Reuss bound will be the same for the rectangular board surrounded with a dashed-dotted line or the triangular domain surrounded wit a dashed line in Figure 2. The most convenient domain for closed-form calculation being the latter, we have:

$\boldsymbol{S}^{R}=\frac{1}{A_{1}+A_{2}}\left(\int_{A_{1}} \boldsymbol{S}(\theta) d A+\int_{A_{2}} \boldsymbol{S}(\theta) d A\right)$,

where $A_{1}$ and $A_{2}$ are triangular sub-domains in Figure 2. Furthermore, the board having always an axis of symmetry ensures that the averaged behavior is orthotropic.

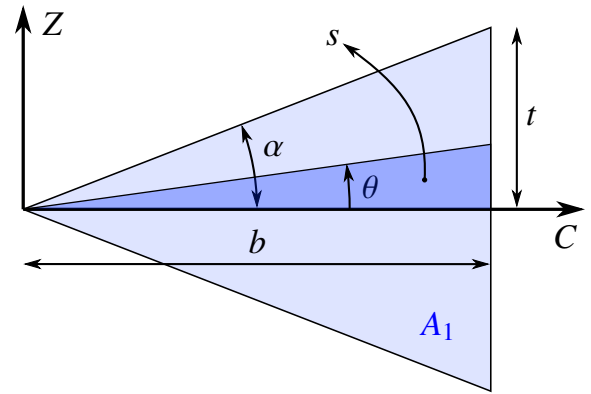

Fig. 3 Integration of mechanical properties on $A_{1}$

In Figure 3, geometrical parameters used for the integration of mechanical properties on the section $A_{1}$ are presented. For an angle $\theta$, the area is $s=\frac{b^{2}}{2} \tan \theta$ and the elementary area is then $d s=\frac{b^{2}}{2} \frac{d \theta}{\cos ^{2} \theta}$. In (4), $S(\theta)$ is expressed as a sum of power function of sinus and cosinus functions weighted by local mechanical properties $S_{i j}$. These functions have to be integrated on the section $A_{1}$ for $\theta \in[-\alpha ; \alpha]$ where $\tan \alpha=t / b$. For example:

$\int_{-\alpha}^{\alpha} \cos ^{4}(\theta) d s=\frac{b^{2}}{2} \int_{-\alpha}^{\alpha} \cos ^{2}(\theta) d \theta=\frac{b^{2}}{2}(\cos \alpha \sin \alpha+\alpha)$.

The integration on $A_{2}$ is similar to the integration on $A_{1}$. From the previous results, it is possible to determine the average $\left\langle\cos ^{4}(\theta)\right\rangle$ on the entire section:

$\left\langle\cos ^{4}(\theta)\right\rangle=\frac{1}{4}\left[3-f^{+}(\alpha)+2 f^{-}(\alpha)\right]$,

where:

$f^{ \pm}(\alpha)=\frac{\alpha}{\tan \alpha} \pm\left(\frac{\pi}{2}-\alpha\right) \tan \alpha$

Proceeding the same way for all functions $s^{4}, c^{2} s^{2}, c^{2}$ and $s^{2}$ and including them in the expression of $\boldsymbol{S}(\theta)$ (4), it is possible to express the Reuss lower bound $S^{R}(\alpha)$ as an orthotropic 
material function of $\alpha$ and local mechanical properties:

$$
\left\{\begin{array}{l}
S_{11}^{R}(\alpha)=S_{11} \\
S_{12}^{R}(\alpha)=\frac{1}{2}\left[S_{12}+S_{13}+\left(S_{12}-S_{13}\right) f^{-}(\alpha)\right] \\
S_{13}^{R}(\alpha)=\frac{1}{2}\left[S_{12}+S_{13}+\left(S_{13}-S_{12}\right) f^{-}(\alpha)\right] \\
S_{22}^{R}(\alpha)=\frac{1}{4}\left[\begin{array}{l}
3\left(S_{22}+S_{33}\right)-2\left(S_{23}+S_{44}\right) \\
+2\left(S_{22}-S_{33}\right) f^{-}(\alpha) \\
+\left(2 S_{23}+2 S_{44}-S_{22}-S_{33}\right) f^{+}(\alpha)
\end{array}\right] \\
S_{33}^{R}(\alpha)=\frac{1}{4}\left[\begin{array}{l}
3\left(S_{22}+S_{33}\right)-2\left(S_{23}+S_{44}\right) \\
+2\left(S_{33}-S_{22}\right) f^{-}(\alpha) \\
+\left(2 S_{23}+2 S_{44}-S_{22}-S_{33}\right) f^{+}(\alpha)
\end{array}\right] \\
S_{23}^{R}(\alpha)=\frac{1}{4}\left[\begin{array}{l}
6 S_{23}+2 S_{44}-S_{22}-S_{33} \\
+\left(S_{22}+S_{33}-2 S_{23}-2 S_{44}\right) f^{+}(\alpha)
\end{array}\right] \\
S_{44}^{R}(\alpha)=\frac{1}{2}\left[\begin{array}{l}
2 S_{23}+4 S_{44}-S_{22}-S_{33} \\
+\left(S_{22}+S_{33}-2 S_{23}-2 S_{44}\right) f^{+}(\alpha)
\end{array}\right] \\
S_{55}^{R}(\alpha)=\frac{1}{2}\left[\begin{array}{l}
\left.S_{55}+S_{66}+\left(S_{55}-S_{66}\right) f^{-}(\alpha)\right] \\
S_{66}^{R}(\alpha)=\frac{1}{2}\left[S_{55}+S_{66}+\left(S_{66}-S_{55}\right) f^{-}(\alpha)\right]
\end{array}\right.
\end{array}\right.
$$

From the expression of the Reuss lower bound of the compliance $S^{R}$, the engineering constants of the associated orthotropic material can be calculated with the following expressions:

$\left\{\begin{array}{c}E_{L}^{R}=\frac{1}{S_{11}^{R}}, E_{C}^{R}=\frac{1}{S_{22}^{R}}, E_{Z}^{R}=\frac{1}{S_{33}^{R}}, \\ v_{L C}^{R}=\frac{S_{12}^{R}}{S_{11}^{R}}, v_{L Z}^{R}=\frac{S_{13}^{R}}{S_{11}^{R}}, v_{C Z}^{R}=\frac{S_{23}^{R}}{S_{22}^{R}}, \\ G_{L C}^{R}=\frac{1}{2 S_{66}^{R}}, G_{L Z}^{R}=\frac{1}{2 S_{55}^{R}}, G_{C Z}^{R}=\frac{1}{2 S_{44}^{R}} .\end{array}\right.$

\subsubsection{Reuss lower bound for a shifted board}

This approach may easily be extended taking into account the distance $z_{1}$ to the pith (Figure 4). The Reuss lower bound of the equivalent layer stiffness of the board delimited by the dashed rectangle in Figure 4 can then be calculated as follows thanks to the additivity of the integral:

$S^{R}\left(\alpha, z_{1}\right)=\frac{z_{2} S^{R}\left(\alpha_{2}\right)-z_{1} S^{R}\left(\alpha_{1}\right)}{t}$.

\subsubsection{Reuss lower bound for a symmetric assembly of shifted boards}

This approach may also be generalized to a symmetric assembly of two boards as presented in Figure 5 with a lateral distance $c_{1}$ to the symmetry axis. This symmetric configuration ensures that the equivalent stiffness is orthotropic in the $(O, L, C, Z)$ frame. Otherwise a coupling - often neglected in engineering practice - would be present between

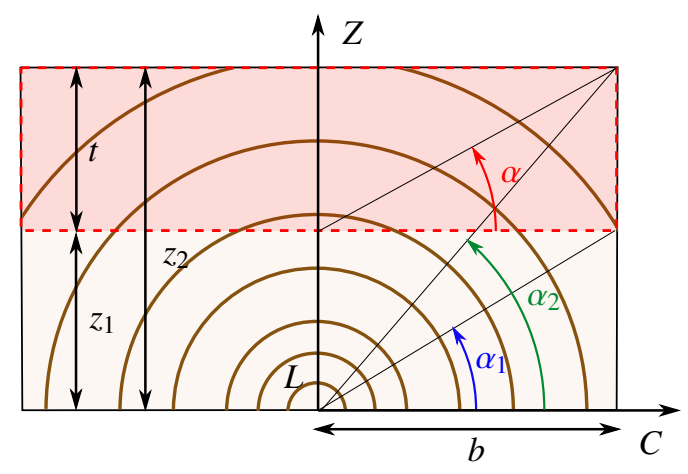

Fig. 4 Scheme of the board section with a distance $z_{1}$ to the pith

shear and normal equivalent stiffnesses. The corresponding Reuss lower bound $S^{R}$ of the symmetric arrangement can be calculated from:

$\boldsymbol{S}^{R}\left(\alpha, z_{1}, c_{1}\right)=\frac{c_{2} z_{2} \boldsymbol{S}^{R}\left(\alpha_{2}\right)-c_{2} z_{1} \boldsymbol{S}^{R}\left(\alpha_{1}\right)-c_{1} z_{2} \boldsymbol{S}^{R}\left(\alpha_{3}\right)+c_{1} z_{1} \boldsymbol{S}^{R}\left(\alpha_{4}\right)}{b t}$.

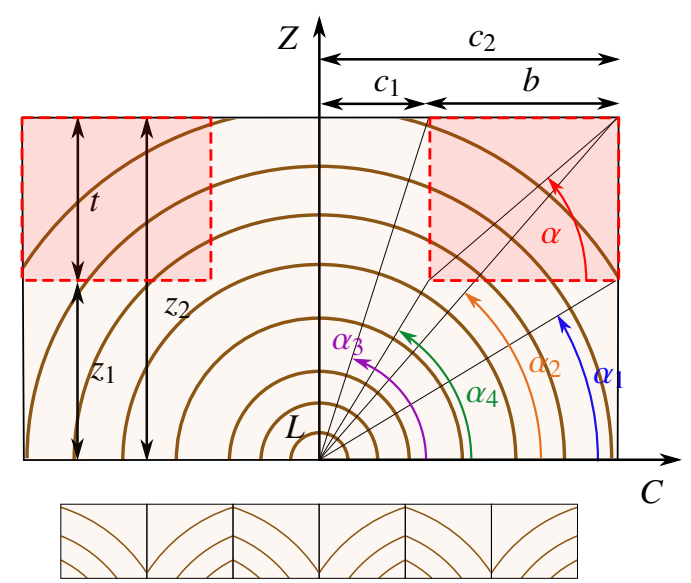

Fig. 5 Scheme of a symmetric assembly of a board section with a position $z_{1} \boldsymbol{e}_{Z}+c_{1} \boldsymbol{e}_{C}$ with respect to the pith

\subsubsection{Further generalizations}

Only configurations leading to an orthotropic equivalent elastic behavior have been presented in detail. It is useful to mention that the present approach may be easily extended to non-orthotropic configurations. Two cases are briefly discussed here.

First, considering only one board in Figure 5 simply requires the derivation of odd contributions in $\theta$ in Equation (4) which do not vanish.

Second, the case of a spiral grained lumber may also be addressed (Figure 6). In such case, the local orientation of the grain follows a helix (frame $(l, R, t)$, rotated by a twist 


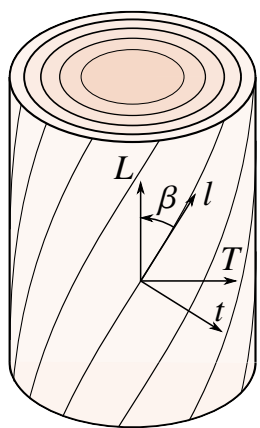

Fig. 6 Local orientation of a spiral grained wood

angle $\beta$ with respect to the ring frame $(L, R, T)$. For a fixed twist angle, the previous approach may be directly applied to the rotated material constants from $(l, R, t)$ to $(L, R, T)$.

\subsubsection{Voigt upper bound}

The same procedure may be followed for deriving the Voigt upper bound of the equivalent stiffness defined as:

$C^{V}=\frac{1}{4 b t} \int_{-b}^{b} \int_{-t}^{t} \boldsymbol{C}(\theta) d C d Z$

Averaging the stiffness matrix is equivalent to assume a uniform strain state $\varepsilon$ over the whole section. Since Kelvin's notation are used, similar expression are found for the stiffness matrix $\boldsymbol{C}^{V}$ of the Voigt upper bound, replacing $\boldsymbol{S}$ by $\boldsymbol{C}$ in (11-18).

As in Section 2.1, the corresponding compliance matrix $S^{V}=\left(C^{V}\right)^{-1}$ is introduced. Then, the engineering constants associated with the Voigt upper bound are calculated from (16) by replacing the superscript $R$ by $V$. Again $S^{V} \neq S^{R}$.

Let us recall that, $\frac{1}{2}^{T} \boldsymbol{\varepsilon} \cdot \boldsymbol{C}^{V} \cdot \boldsymbol{\varepsilon}$ is an upper bound of the potential energy of the board for any average strain $\varepsilon$ and $\frac{1}{2} \sigma \cdot S^{R} \cdot \sigma$ is an upper bound of the complementary energy for any average stress $\sigma$. From Legendre-Fenchel transform applied to the potential energy, it is possible to prove:

$\forall \sigma, \quad{ }^{T} \sigma \cdot\left(S^{V}\right)^{-1} \cdot \sigma \leq{ }^{T} \sigma \cdot S^{H} \cdot \sigma \leq{ }^{T} \sigma \cdot S^{R} \cdot \sigma$

where $S^{H}$ is the equivalent compliance tensor to be estimated. Considering for instance a state of pure traction in direction $L:{ }^{T} \boldsymbol{\sigma}=(1,0,0,0,0,0)$ in (20) leads to:

$\frac{1}{E_{L}^{V}} \leq \frac{1}{E_{L}^{H}} \leq \frac{1}{E_{L}^{R}} \quad$ or $\quad E_{L}^{R} \leq E_{L}^{H} \leq E_{L}^{V}$

which illustrates that Young's moduli as well as shear moduli of the equivalent stiffness are bounded by those of the Reuss lower bound and the Voigt upper bound.

Although relatively cumbersome, there is no difficulty in the application of the method and the evaluation of the various bounds is straightforward from the material local properties as will be shown in the next section.

\section{Equivalent-layer stiffness of Norway-spruce with glued narrow edges}

An application of preceding formulas is made to estimate the equivalent layer stiffness of boards with glued edges from the local behavior at ring scale measured by Keunecke et al $(2007,2008)$ on Norway spruce (Table 1). Only sawing patterns of boards including an axis of symmetry are investigated (17).

\subsection{Equivalent Young's moduli}

\subsubsection{Equivalent longitudinal Young's modulus $E_{L}$}

The equivalent longitudinal Young's modulus $E_{L}$ is an important characteristic of the CLT behavior particularly under bending and under in-plane load. The Reuss bound $E_{L}^{R}$ of the equivalent longitudinal modulus is equal to the local longitudinal modulus $E_{L}$ for any sawing pattern (see 16). The equivalent longitudinal modulus $E_{L}^{V}$ estimated numerically from the Voigt upper bound is almost equal to the Reuss bound for varying sawing patterns: the relative difference is lower than $0.2 \%$ for $\frac{2 b}{t}<20$ and $\frac{z_{1}}{2 b}<1.5$. This is a consequence of the high stiffness contrast between $E_{L}$ and $E_{R}$ and $E_{T}$. Thus, the sawing pattern of boards is indifferent in practice regarding the equivalent longitudinal Young's modulus $E_{L}$ of CLT with glued edges.

\subsubsection{Equivalent normal and cross Young's moduli $E_{Z}$ and $E_{C}$}

The equivalent normal Young's modulus $E_{Z}$ has only a limited role in the behavior of CLT panels: it plays a role mostly when the CLT panel is placed between two vertical elements and is locally punched. Figure 7 shows $E_{Z}$ calculated from Reuss and Voigt bounds for a varying board aspect ratio $\frac{2 b}{t}$ and for a varying relative distance to the pith $\frac{z_{1}}{2 b}$. It is observed that $E_{Z}$ varies strongly between $620 \mathrm{MPa}$ and $230 \mathrm{MPa}$ which is approximately one third of the local radial modulus $E_{R}$. It is minimum for a relative distance to the pith $\frac{z_{1}}{2 b}$ between 0.2 and 0.4 and a board aspect ratio $\frac{2 b}{t}>4$ which corresponds to common sawing patterns used for cross-layer. Using directly local moduli $E_{R}$ and $E_{T}$ in applications may thus overestimate the equivalent modulus from $200 \%$ to $274 \%$. Similar conclusions were drawn in previous studies based on FEA (Nairn, 2007; Gustafsson and Danielsson, 2013).

The equivalent cross Young's modulus $E_{C}$ is involved in the calculation of the bending and membrane stiffness. In Figure 7, the equivalent cross Young's modulus $E_{C}$ calculated from Reuss and Voigt bounds is plotted for a varying board aspect ratio $\frac{2 b}{t}$ and for a varying relative distance to the pith $\frac{z_{1}}{2 b}$. It is observed that the equivalent cross Young's modulus $E_{C}$ varies strongly with the sawing pattern. It can 

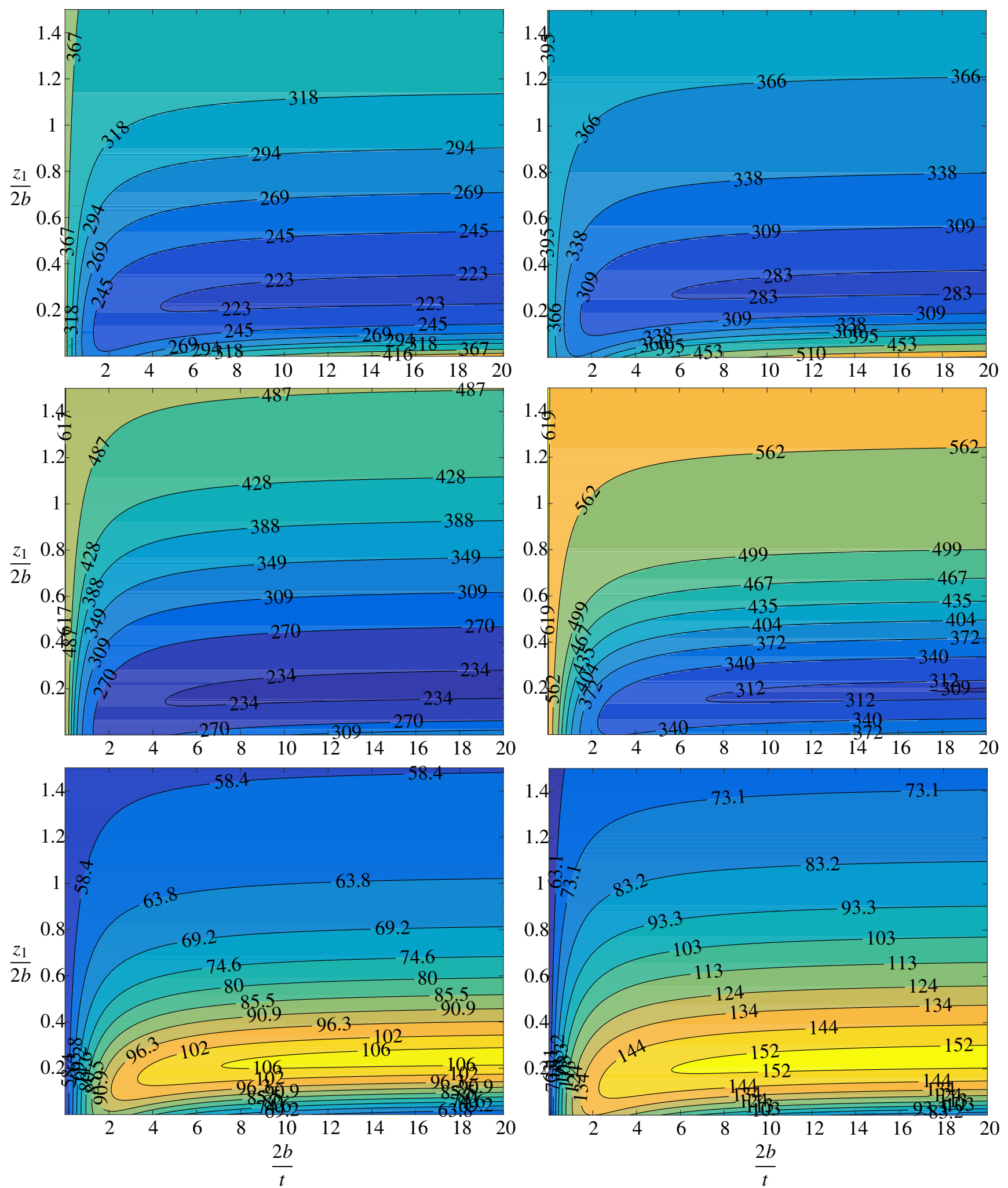

Fig. 7 Reuss lower bound (left) and Voigt upper bound (right) for the estimation of $E_{C}$ (top), $E_{Z}$ (middle) and $G_{C Z}$ (bottom), isolines in MPa 


\begin{tabular}{ccrrrrrrrr}
\hline & $E_{L}$ & $E_{R}$ & $E_{T}$ & $G_{L R}$ & $G_{L T}$ & $G_{R T}$ & $v_{L R}$ & $v_{L T}$ & $v_{R T}$ \\
\hline Value (MPa) & 12800 & 625 & 397 & 617 & 587 & 53 & 0.36 & 0.45 & 0.48 \\
CoV (\%) & 9.2 & 20.4 & 10.3 & 12.1 & 10.2 & 10.9 & 13.2 & 8.2 & 19.2 \\
No. of specimen & 10 & 13 & 11 & 120 & 120 & 120 & 10 & 10 & 13 \\
\hline
\end{tabular}

Table 1 Elastic Properties of Norway spruce (Keunecke et al, 2007, 2008)

be equal to $220 \mathrm{MPa}$ which is almost three times lower than the local Young's modulus $E_{R}$. This value is in contradiction with what is suggested in current recommendations for a revised version of the Eurocode 5.1.1 (Eurocode 5-1-1, 2015) where a value of $450 \mathrm{MPa}$ is suggested which could underestimate deformations due to cross layers. Note, however, that $E_{C}$ is generally assumed negligible compared to $E_{L}$ in the calculation of the bending and membrane stiffness of CLT.

In Figure 8, the equivalent cross and normal Young's moduli $E_{C}^{R}, E_{C}^{V}, E_{Z}^{R}$ and $E_{Z}^{V}$ are compared to the compliance average $E_{N}^{S}$ and the stiffness average $E_{N}^{C}$. As expected, for $\alpha=\arctan \frac{t}{b}$ close to $0^{\circ}$ and $90^{\circ}$, the bounds tend to the local behavior since the ring orientation is almost uniform in the section: local orientations coincide with the global orientations. It is further observed that, for $\alpha$ between $30^{\circ}$ and $60^{\circ}$ which corresponds to practical values of the aspect ratio, the equivalent Young's moduli of Voigt and Reuss bounds can be approximated directly by the moduli from averages of the stiffness $E_{N}^{C}$ and of the compliance $E_{N}^{S}$.

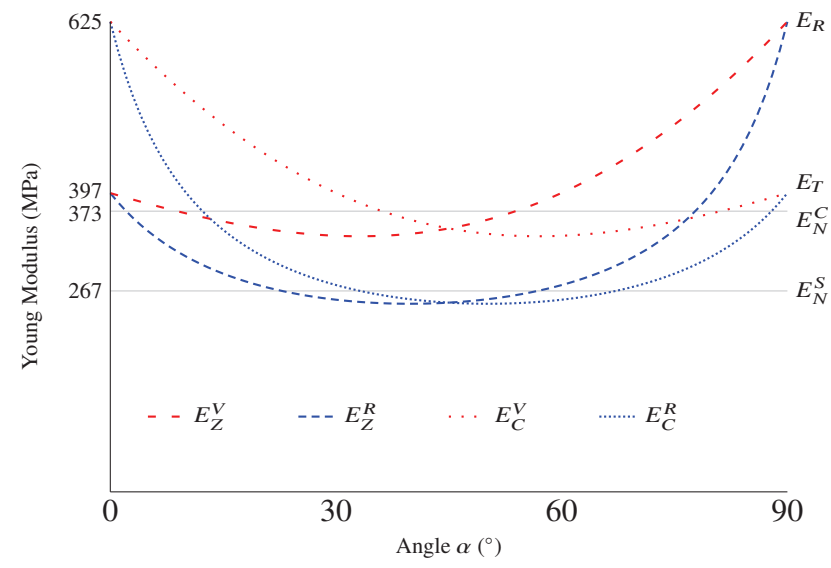

Fig. 8 Young's moduli of Voigt and Reuss bounds for $z_{1}=0$

\subsubsection{Poisson's ratio $v_{L C}$ and $v_{C L}$}

In current recommendations, the bending stiffness $D$ of longitudinal layers in CLT is considered proportional to $E_{L}$ and not to the plane-strain Young's modulus $\frac{E_{L}}{1-v_{L C} v_{C L}}$ as should be done in Classical Lamination Theory. From Reuss and Voigt bounds, the Poisson's ratios product $v_{L C} v_{C L}$ varies between 0.003 to 0.007 . Thus, the assumption of $E_{L} \approx$ $\frac{E_{L}}{1-v_{L C} v_{C L}}$ is valid for CLT panels.

\subsection{Equivalent shear moduli}

\subsubsection{Equivalent longitudinal-layer and in-plane shear moduli $G_{L Z}$ and $G_{L C}$}

From calculations, for varying board aspect ratio $\frac{2 b}{t}$ and relative distance to the pith $\frac{z_{1}}{2 b}$, the gap between bounds of the longitudinal-layer shear modulus $G_{L Z}$ and the in-plane shear modulus $G_{L C}$ is less than $0.1 \%$. Indeed, because local shear moduli $G_{L R}=617 \mathrm{MPa}$ and $G_{L T}=587 \mathrm{MPa}$ are rather close, $G_{L Z}$ and $G_{L C}$ vary slightly between these values. This might not be the case for other species, and larger variations may be be observed. Finally, it is not surprising that the stiffness and compliance averages $G_{N N}^{S} \approx G_{N N}^{C}=602 \mathrm{MPa}$ appears as good approximations of $G_{L R}$ and $G_{L T}$ for CLT with glued narrow edges.

\subsection{Equivalent cross-layer shear stiffness modulus $G_{C Z}$}

In Figure 7, Reuss and Voigt bounds of equivalent layer shear stiffness modulus $G_{C Z}$ are plotted for varying board aspect ratios $\frac{2 b}{t}$ and for relative distance to the pith $\frac{z_{1}}{2 b}$. It is observed that, if the board aspect ratio is large enough, there is a sawing pattern, corresponding to a relative distance to the pith $0.2<\frac{z_{1}}{2 b}<0.3$, which maximizes $G_{C Z}$. For these boards, the equivalent cross layer shear stiffness modulus lies between 100 and $150 \mathrm{MPa}$ approximately which is twice to three times higher than the local rolling shear stiffness modulus $G_{R T}$. Hence, the present closed-form bounds confirm previous observations obtained from experiments as well as numerical simulations (Aicher and Dill-Langer, 2000; Jakobs, 2005; Perret et al, 2018; Ehrhart and Brandner, 2018).

In this section, upper and lower bounds were calculated for boards with glued edges. These bounds are also valid for any assembly of identical boards such as GLT. Nevertheless, most of CLT manufacturers do not glue narrow edges. Hence, free narrow edges effects, which are specific to CLT, are discussed in the following section. 


\section{Influence of free narrow edges on the cross-layer shear stiffness modulus $G_{C Z}$}

\subsection{Influence of free narrow edges}

In this section, the impact of free narrow edges on the global behavior of CLT compared to results of Section 3 is discussed. The free narrow edges boundary condition imposes $\sigma_{C C}=\sigma_{L C}=\sigma_{C Z}=0$ on narrow edges. Since a uniform strain is assumed for the Voigt upper bound calculation, it is also an upper bound when narrow edges are free. In contrast, these conditions are not always compatible with the uniform stress assumption in the Reuss lower bound calculation. The longitudinal Young's modulus $E_{L}^{R}$, the normal Young's modulus $E_{Z}^{R}$ and the longitudinal shear modulus $G_{L Z}^{R}$ are calculated assuming uniform stress $\sigma_{L L}, \sigma_{Z Z}$ and $\sigma_{L Z}$ which are compatible with the free narrow edges boundary condition. Hence, $E_{L}^{R}, E_{Z}^{R}$ and $G_{L Z}^{R}$ are also lower bounds of the corresponding homogenized engineering constants for CLT with free narrow edges and the results obtained in the previous section still hold for these moduli. Nonetheless, the equivalent cross Young's modulus $E_{C}^{R}$, the equivalent in-plane shear modulus $G_{L C}^{R}$ and the equivalent cross-layer shear modulus $G_{C Z}^{R}$ are calculated from an assumption of uniform stress $\sigma_{C C}, \sigma_{L C}$ and $\sigma_{C Z}$ over the whole board. Hence, $E_{C}^{R}, G_{L C}^{R}$ and $G_{C Z}^{R}$ are no lower bounds of the corresponding homogenized engineering constants for CLT with free narrow edges. Actually these moduli are involved in more complex plate behavior of CLT which are now briefly reviewed.

Even if $G_{L Z}$ and $G_{L C}$ have similar values for glued narrow edges, they are involved in different plate mechanisms: $G_{L Z}$ contributes to the global out-of-plane shear behavior whereas $G_{L C}$ plays a central role in the in-plane shear behavior of CLT panels. For CLT with free narrow edges, the in-plane shear and torsion behavior, depending on $G_{L C}$, is more complex than for glued edges. Indeed, in-plane shear stress cannot be transferred directly to the neighboring board because of free edges (Silly, 2010). The behavior is thus intrinsically 3D and stiffness can only be estimated considering a superposition of layers. In particular, Moosbrugger et al (2006) studied the in-plane shear behavior of an infinitely thick CLT with regular gaps between neighboring boards. They observed a significant decrease in the apparent in-plane shear modulus $G_{L C}$ up to half the one estimated for boards with glued edges. These results were compared later with 3-ply and 5-ply CLT by Bogensperger et al (2010). Following Hashin $(1986,1987)$ variational principles, Nairn (2017) also suggested an equivalent layer in-plane shear modulus. Finally, Franzoni et al $(2017,2018)$ also studied this behavior at the scale of a CLT plate with small or large gaps by means of a periodic plate homogenization scheme and suggested closed-form formula.
Similarly to the in-plane shear behavior, a 3D study is necessary for the equivalent cross Young's modulus $E_{C}$ since stress cannot be transmitted directly from one board to the other (Nairn, 2017). However, this mechanical behavior was less investigated as this stiffness and strength direction is often neglected in practice.

Finally, the detailed treatment of the cross layer shear stiffness $G_{C Z}$ requires also a 3D analysis as it involves complex interactions with the upper and lower longitudinal layers. Neglecting the influence of the sawing pattern, this analysis was achieved by Franzoni et al $(2017,2018)$ and closed-form formula were suggested as well. This required a modeling based on a thick plate homogenization scheme (Lebée and Sab, 2012b; Sab and Lebée, 2015).

\subsection{Numerical upper bounds for the cross-layer shear} stiffness modulus $G_{C Z}$

In order to compare the suggested bounds for the equivalent cross layer shear stiffness modulus $G_{C Z}$ with existing experimental data available in the literature, a simpler and approximate approach is followed in this section.

Indeed, because of the stiffness contrast between the longitudinal layers and the cross layers it is possible to argue that the cross layer is mostly sheared by the longitudinal layers (Lebée and Sab, 2012a). Hence in practice the equivalent cross layer shear modulus is mostly determined experimentally from single lap shear tests (Ehrhart et al, 2015; Ehrhart and Brandner, 2018) where narrow edges are free. Following this global picture, a numerical finite elements study is achieved in order to compute an upper bound of the equivalent cross-layer shear stiffness modulus $G_{C Z}$ with glued and free narrow edges. Several similar studies have already been performed (Aicher and Dill-Langer, 2000; Jakobs, 2005) but only for free narrow edges.

It is assumed that the cross-layer is sheared by the relative displacement of the adjacent longitudinal layer. Since the displacement is imposed, the cross-layer shear stiffness modulus $G_{C Z}^{\text {num }}$ calculated numerically is an upper bound of $G_{C Z}$. This upper bound is more precise than Voigt upper bound since displacement conditions are imposed only on the boundary whereas a uniform shear strain field is imposed in the whole board for the Voigt upper bound. However, when narrow edges are free, the estimation from this upper bound may be far from the exact value since no lower bound is available. This may appear when the aspect ratio of the board is small and will be discussed in the next section.

Note that, in Aicher and Dill-Langer (2000) and Jakobs (2005), the cross-layer was modeled as well as the longitudinal adjacent layer (Figure 9). Because of the stiffness contrast between layers, this approach is almost equivalent to imposing directly displacement conditions on the upper 
and lower faces of the cross-layer. Indeed, Lebée and Sab (2012a) demonstrated that it is possible to isolate the core of a sandwich (corresponding here to the cross-layer), from the skins (here the longitudinal layers) and to obtain an upper bound of the cross-layer shear stiffness by imposing a relative displacement between upper and lower faces.

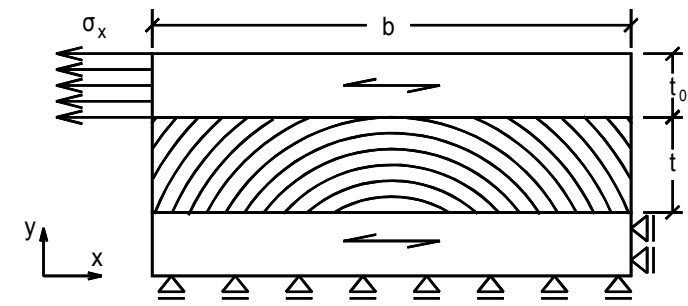

Fig. 92 D numerical simulation for calculation of the equivalent crosslayer shear stiffness (Aicher and Dill-Langer, 2000)

The cross-layer is assumed to be periodic with an alternative orientation of the sawing patterns (Figure 10). Hence, for glued narrow edges, a representative volume element (RVE) can be modeled as two adjacent boards. It is then possible to consider symmetry to reduce the problem to only two half board sections (Figure 10) called Sub-Representative Volume Element (SRVE). Consequently, symmetry conditions are imposed on lateral sections for a board with glued edges (Figure 11). For free edges, only half a board is modeled with a free boundary condition and a symmetry condition as shown in Figure 12. For each board, the local orientation of timber is varying according to the given sawing pattern. Finally, because the problem is invariant in direction $L$ and symmetric with respect to any plane of normal $\boldsymbol{e}_{L}$, only a 2D numerical simulation is required with in-plane deformations.

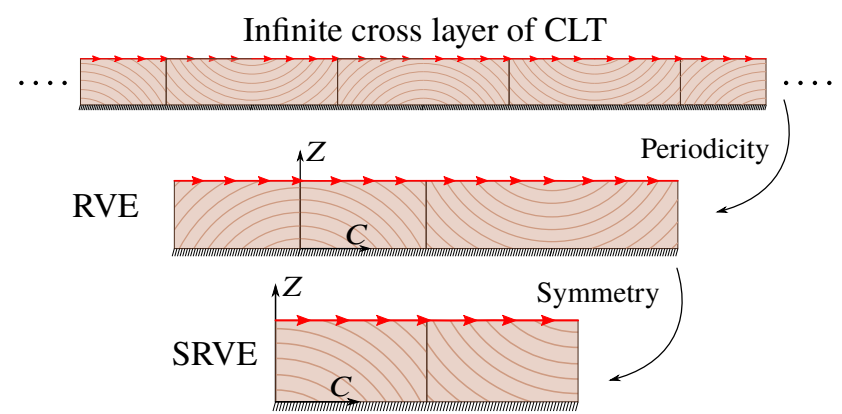

Fig. 10 Simplification of the model thanks to periodicity and symmetries

The equivalent cross-layer shear stiffness modulus $G_{C Z}$ can be estimated from the strain energy in the board $W_{\text {ela }}$ :

$W_{\text {ela }}=\frac{1}{2} G_{C Z} \gamma^{2} b t$,

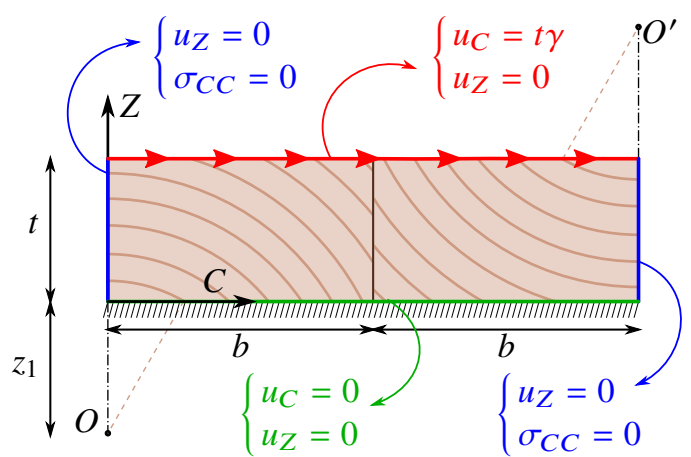

Fig. 11 2D SRVE for the calculation of the equivalent cross-layer shear stiffness of CLT with glued edges

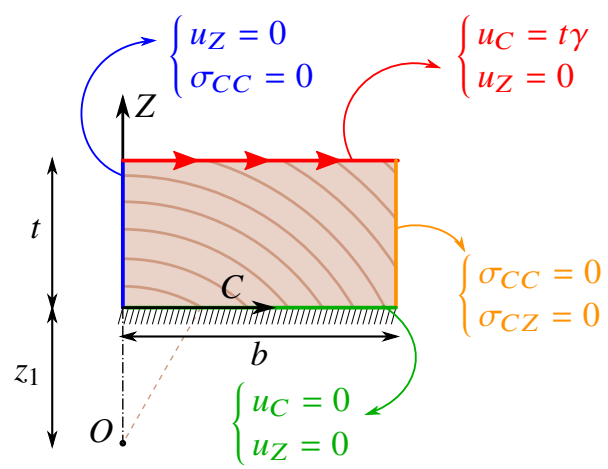

Fig. 12 2D SRVE for the calculation of the equivalent cross-layer shear stiffness of CLT with free edges

where $\gamma=2 \varepsilon_{L C}=\frac{u_{C}}{t}$ is the averaged shear strain in the specimen.

\subsection{Comparison with experimental data}

In this section, closed-form as well as numerical bounds are compared to direct measurements of the equivalent crosslayer shear stiffness modulus $G_{C Z}$ found in the literature. As in Section 3, all theoretical values are derived from the local stiffness measured by Keunecke et al $(2007,2008)$ on Norway spruce (Table 1) whereas the data for the direct measurement comes from other samples. This may explain some discrepancies in addition to wood natural variability.

\subsubsection{Data from Franzoni et al (2016a) and Perret et al (2018)}

Franzoni et al (2016a) performed double lap shear tests on a small batch of CLT boards whose typical cross section is given in Figure 1. This corresponds approximately to $z_{1}=t / 10$. Perret et al (2018) performed 4-point bending on sandwich beams on the same batch. Hence, in Franzoni et al (2016a) tests, narrow edges were free, whereas in Perret et al (2018) tests, narrow edges were glued. Because of the small number of different boards, the variability observed 
is not statistically representative of the species but allows qualitative observations.

In Figure 13, predicted and measured values of the equivalent cross-layer shear stiffness modulus $G_{C Z}$ are plotted for $\alpha \in\left[0^{\circ}, 90^{\circ}\right]$ and $z_{1}=t / 10$. The closed-form Reuss lower bound and Voigt upper bound are plotted as well as both numerical upper bounds for free and glued narrow edges.

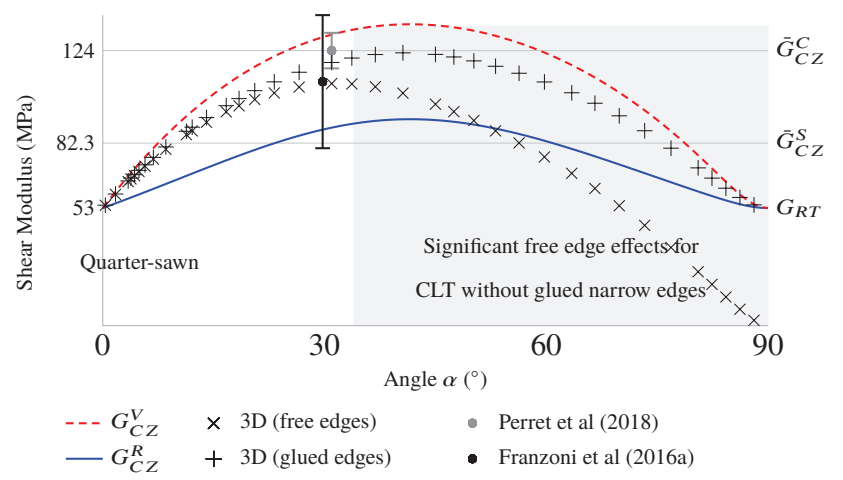

Fig. 13 Equivalent cross-layer shear moduli as a function of $\alpha$ for $z_{1}=t / 10$

As already noted in Section 3, $G_{C Z}$ may be up to 2.5 times larger than the local rolling shear modulus $G_{R T}$. In addition, for $30^{\circ}<\alpha<60^{\circ}$, the closed-form bounds $G_{C Z}^{R}$ and $G_{C Z}^{V}$ can be well approximated by direct averages $G_{N N}^{S}$ and $G_{N N}^{C}$ derived in Section 2.1.

As expected, both numerical upper bounds are lower than the closed-form upper bound. The free narrow edge upper bound is lower than the glued narrow edge bound as well. This will be the case for all further comparisons. For small angle $\alpha$, numerical upper bounds for glued and unglued narrow edges are similar. Indeed in this range of aspect ratio, the boards are thin and free edges effects are negligible. Nevertheless, for $\alpha>30^{\circ},(2 b / t<4)$, the difference between free and glued narrow edges numerical upper bounds is becoming significant as the free edges bound is dropping rapidly (recall that the closed-form lower bound is only valid for glued edges). Hence for aspect ratios smaller than 4 , the closed-form bound should be used with care and more refined approaches are required for estimating the cross-layer shear stiffness modulus, such as periodic plate homogenization techniques (Sab and Lebée, 2015; Franzoni et al, 2017), taking annual rings orientation into account.

Finally, considering wood variability, the experimental data is in agreement with the theoretical predictions. A slightly higher value was measured by Perret et al (2018) since tests were performed with glued narrow edges contrary to Franzoni et al (2016a).

\subsubsection{Data from Ehrhart et al (2015)}

Ehrhart et al (2015) performed single lap shear tests on boards with three different aspect ratios and three relative distances to the pith. In this study, the sampling is broader and boards were tested individually with free narrow edges.

In Figure 14, cross-layer shear moduli are plotted as a function of the angle $\alpha$ for a fixed relative distance to the pith $\frac{z_{1}}{t}=1.5$. Closed-form bounds, numerical upper bounds and the recommendations from Ehrhart et al (2015) and Schickhofer et al (2016) are compared to the experimental data from Ehrhart et al (2015). The prediction given by theoretical bounds is in very good agreement with experimental data and provides a slightly finer description of the influence of the aspect ratio on the cross-layer shear stiffness modulus than current recommendations. Again, for $\alpha>20^{\circ}$

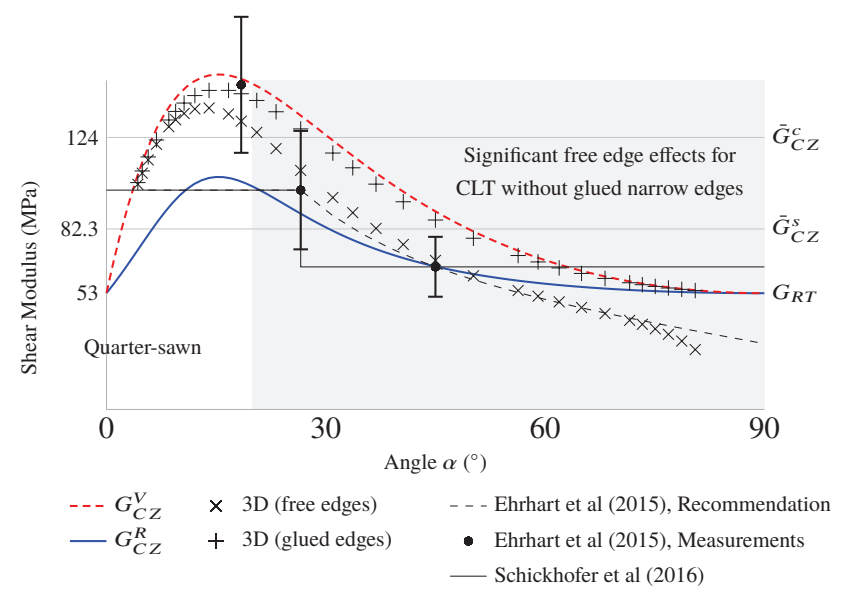

Fig. 14 Equivalent cross-layer shear moduli as a function of $\alpha$ for $z_{1}=1.5 t$

$(2 b / t<6)$, the difference between free and glued narrow edges numerical upper bounds is becoming significant.

In Figure 15, the influence of the relative distance to the pith $\frac{z_{1}}{t}$ is investigated for an angle $\alpha=26.6^{\circ}(2 b / t=4)$. This aspect ratio corresponds to the limit value where Schickhofer et al (2016) recommend to drop the cross-layer shear stiffness from $100 \mathrm{MPa}$ to $65 \mathrm{MPa}$. For this aspect ratio, the difference between glued and free narrow edges numerical upper bounds is rather large (about 20\%) showing already important free edges effects. Experimental data is globally in agreement with theoretical predictions except for boards furthest to the pith which yielded a rather low measured shear stiffness. No reasonable explanation of this lower value was found. 


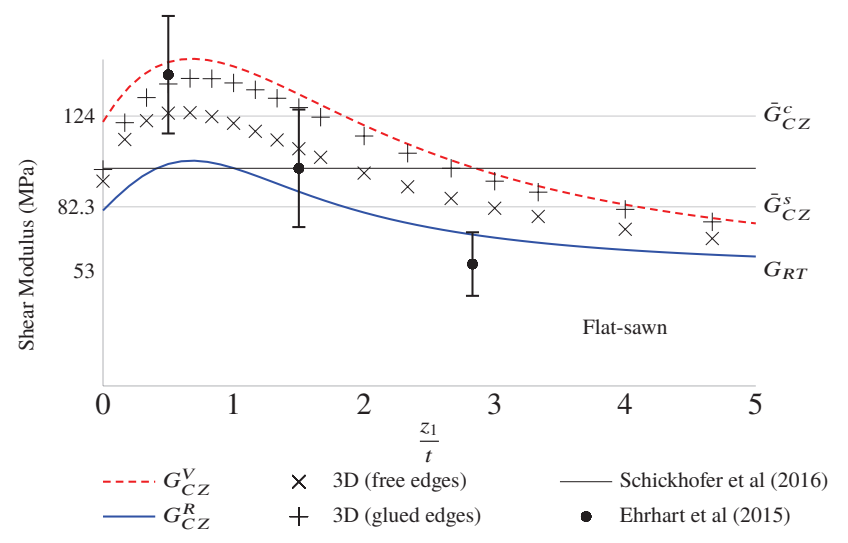

Fig. 15 Equivalent cross-layer shear moduli as a function of $z_{1}$ for $\alpha=26.6^{\circ}$

\section{Conclusion}

Closed-form upper and lower bounds were derived to estimate the equivalent stiffness of timber from averages over the board section for varying sawing pattern. These bounds are relevant for estimating the equivalent layer stiffness of CLT panel with glued narrow edges.

From these results, it was observed that the Young's longitudinal modulus $E_{L}$ is almost constant for varying sawing patterns and can be estimated from usual local tests. Furthermore, the cross and normal Young's moduli $E_{C}$ and $E_{Z}$ may decrease strongly compared to local stiffness moduli and could take lower values than those suggested in the revised version of the Eurocode 5.1.1 (Eurocode 5-1-1, 2015).

It was also observed that for varying sawing patterns, the in-plane shear modulus $G_{L C}$ and the longitudinal shear modulus $G_{L Z}$ have almost the same value for glued narrow edges. Nevertheless, it is well established that they are involved in very different mechanism regarding CLT when narrow edges are free. More specifically, $G_{L Z}$ is a priori not influenced by free narrow edges conditions whereas a significant reduction of $G_{L C}$ is observed between boards with glued and free narrow edges. This observation is in contradiction with what is recommended in the revised version of the Eurocode 5.1.1 (Eurocode 5-1-1, 2015) where no distinction is made between these two parameters.

Finally, the equivalent cross-layer shear stiffness $G_{C Z}$ varies strongly with the sawing pattern. With a careful choice of boards, it may be equal to $100 \mathrm{MPa}$ to $150 \mathrm{MPa}$. Whereas for glued narrow edges, the bounds yield very relevant estimate of $G_{C Z}$, a significant impact of free narrow edges was observed from numerical simulations. For boards with aspect ratio $2 b / t$ smaller than 4 , the suggested bounds may be used for identifying trends but not as predictive formulas.

In terms of CLT design, closed-form formulas confirm the common intuition that having growth ring oriented $45^{\circ}$ is favorable in terms of cross-layer shear stiffness. This suggests that a careful alternation of quarter sawn boards may yield significantly stiffer layers. The second advantage of having closed-form formula is the possibility to perform sensitivity analysis as well as reliability analysis which is critical with bio-based materials.

\section{Conflict of interest}

On behalf of all authors, the corresponding author states that there is no conflict of interest.

\section{References}

Aicher S, Dill-Langer S (2000) Basic considerations to rolling shear modulus in wooden boards. Tech. rep., Otto Graf Journal

Aicher S, Dill-Langer G, Höfflin L (2001) Effect of Polar Anisotropy of Wood Loaded Perpendicular to Grain. Journal of Materials in Civil Engineering 13(1):2-9

Aicher S, Christian Z, Hirsch M (2016) Rolling shear modulus and strength of beech wood laminations. Holzforschung 70(8):773-781

Bogensperger T, Moosbrugger T, Silly G (2010) Verification of CLT-plates under loads in plane. 11th World Conference on Timber Engineering 2010, WCTE 2010 1:231-240

Ehrhart T, Brandner R (2018) Rolling shear: Test configurations and properties of some European soft- and hardwood species. Engineering Structures 172(tba):554-572

Ehrhart T, Brandner R, Schickhofer G, Frangi A (2015) Rolling Shear Properties of some European Timber Species with Focus on Cross Laminated Timber (CLT): Test Configuration and Parameter Study. In: Proceedings of the 2nd International Network on Timber Engineering Research meeting, vol 48, pp 61-78

Eurocode 5-1-1 (2004) EN 1995 1-1: Design of timber structures. Part 1-1: General -Common rules and rules for buildings. European Committee for Standardization, CEN, Bruxelles, Belgium

Eurocode 5-1-1 (2015) Working draft of design of CLT in a revised Eurocode 5-1-1. Version 2015-10-30 (confidential). European Committee for Standardization, CEN, Bruxelles, Belgium

Franzoni L, Lebée A, Lyon F, Forêt G (2016a) Bending behavior of regularly spaced CLT panels. In: WCTE 2016 - World Conference on Timber Engineering

Franzoni L, Lebée A, Lyon F, Foret G (2016b) Influence of orientation and number of layers on the elastic response and failure modes on CLT floors: modeling and parameter studies. European Journal of Wood and Wood Products 74(5):671-684

Franzoni L, Lebée A, Lyon F, Forêt G (2017) Elastic behavior of Cross Laminated Timber and timber panels with regular 
gaps: Thick-plate modeling and experimental validation. Engineering Structures 141:402-416

Franzoni L, Lebée A, Lyon F, Forêt G (2018) Closed-form solutions for predicting the thick elastic plate behavior of CLT and timber panels with gaps. Engineering Structures 164:290-304

Gagnon S, Pirvu C (2013) CLT handbook: Cross Laminated Timber. FPInnovations, Québec, Canada

Gustafsson PJ, Danielsson H (2013) Perpendicular to grain stiffness of timber cross sections as affected by growth ring pattern, size and shape. European Journal of Wood and Wood Products 71(1):111-119

Hashin Z (1986) Analysis of stiffness reduction of cracked cross-ply laminates. Engineering Fracture Mechanics 25(5-6):771-778

Hashin Z (1987) Analysis of Orthogonally Cracked Laminates Under Tension. Journal of Applied Mechanics 54(4):872

Jakobs A (2005) Zur Berechnung von Brettlagenholz mit starrem und nachgiebigem Verbund unter plattenartiger Belastung mit besonderer Berücksichtigung des Rollschubes und der Drillweichheit [On the calculation of Cross Laminated Timber as a composite plate with special consideration on the rolling-shear and the in-plane shear stiffness]. $\mathrm{PhD}$ thesis, Universität der Bundeswehr München

Keunecke D, Sonderegger W, Pereteanu K, Lüthi T, Niemz P (2007) Determination of Young's and shear moduli of common yew and Norway spruce by means of ultrasonic waves. Wood Science and Technology 41(4):309-327

Keunecke D, Hering S, Niemz P (2008) Three-dimensional elastic behaviour of common yew and Norway spruce. Wood Science and Technology 42(8):633-647

Kreuzinger H (1999) Plate and shell structures. A model for common calculation tools (in German). Bauen mit Holz 1:34-39

Lebée A, Sab K (2012a) Homogenization of cellular sandwich panels. Comptes Rendus Mécanique 340(4-5):320337

Lebée A, Sab K (2012b) Homogenization of thick periodic plates: Application of the Bending-Gradient plate theory to a folded core sandwich panel. International Journal of Solids and Structures 49(19-20):2778-2792

Lomholt I (2009) Stadthaus London : Murray Grove Building, http://nbn-resolving.de/urn:nbn:de:bvb:706-1363

Mairs J (2016) Anders Berensson proposes wooden skyscraper with decorative facade for Stockholm, https://www.dezeen.com/2016/04/25/anders-berenssonarchitects-tratoppen-wooden-skyscraper-conceptstockholm-cross-laminated-timber/

Moosbrugger T, Guggenberger W, Bogensperger T (2006) Cross Laminated Timber wall segments under homogeneous shear with and without openings. In: WCTE 2006, 9th World Conference on Timber Engineering, pp 1-10

Nairn JA (2007) A numerical study of the transverse modulus of wood as a function of grain orientation and properties. Holzforschung 61(4):406-413

Nairn JA (2017) Cross laminated timber properties including effects of non-glued edges and additional cracks. European Journal of Wood and Wood Products 75(6):973-983

Perret O, Lebée A, Douthe C, Sab K (2016) The Bending-Gradient theory for the linear buckling of thick plates: Application to Cross Laminated Timber panels. International Journal of Solids and Structures 87:139-152

Perret O, Lebée A, Douthe C, Sab K (2018) Experimental determination of the equivalent-layer shear stiffness of CLT through four-point bending of sandwich beams. Construction and Building Materials 186:1132-1143

Sab K, Lebée A (2015) Homogenization of Thick and Heterogeneous Plates. Wiley-ISTE

Schickhofer G, Brandner R, Bauer H (2016) Introduction to CLT, Product Properties, Strength Classes. In: Cross laminated Timber - a competitive wood product for visionary and fire safe buildings: Joint Conference of COST Actions FP1402 and FP1404

Silly G (2010) Numerical study on in-plane shear and torsional stiffness of Cross Laminated Timber (in German). Diplomarbeit, Graz University of Technology

Thiel A, Krenn H (2016) Buckling loads for cross-laminated timber elements under uniaxial in-plane compression. In: Proceedings of the 14th World Conference on Timber Engineering, Vienna (AUT)

Thiel A, Schickhofer G (2010) CLTdesigner - a software tool for designing Cross Laminated Timber elements: 1Dplate-design. In: World Conference on timber engineering Thiel A, Schickhofer G (2013) ULS and SLS design of CLT and its implementation in the CLT designer. In: Schickhofer G, Harris R, Ringhofer A (eds) Focus Solid Timber Solution - European Conference on Cross Laminated Timber (CLT) 